\title{
Adding extended-release methylphenidate to psychological intervention for treatment of methamphetamine dependence: A double-blind randomized controlled trial
}

\author{
Alireza Noroozi ${ }^{1,2}$, Seyed Abbas Motevalian³, Mohammad-Reza Zarrindast ${ }^{2,4,5,6,7}$, Javad Alaghband-Rad ${ }^{8}$, \\ Shahin Akhondzadeh*1,9 (1D)
}

Received: 12 May 2019

Published: 14 Oct 2020

\section{Abstract}

Background: Iran has been faced with an emerging epidemic of methamphetamine (MA) use during recent years. No effective pharmacotherapy has been identified for MA treatment; and psychological interventions are the only available effective treatment. The aim of this study is to investigate the efficacy and safety of extended-release methylphenidate (ER-MTP) for the treatment of methamphetamine dependence.

Methods: Sixty-two people with methamphetamine dependence, according to DSM-IV-TR, were randomly assigned to either fixeddose extended-release methylphenidate (ER-MTP) (60 mg per day) or placebo for 12 weeks. All participants received twice-weekly cognitive behavioral treatment for stimulant dependence. Recent drug use and craving level were measured using weekly rapid urine test and craving visual analogue scale, respectively. The severity of addiction was measured using the Addiction Severity Index at baseline and study completion. Assessment of MA withdrawal was conducted using Amphetamine Withdrawal Questionnaire and Amphetamine Selective Severity Assessment at baseline, day 3, week 1, week 4 and week 12. Depression and high-risk behaviors assessed with the Beck Depression Inventory and the high-risk behavior questionnaire at baseline, weeks 4 and 12 of the study. SPSS software version 22 was used for data analysis and $\mathrm{p}<0.05$ was considered significant.

Results: Percent of weekly MA negative urine tests was not significantly different between groups during the course of the study $(p=0.766)$. Two groups showed similar retention rates. Changes in MA craving, withdrawal, addiction severity, depression and high-risk behaviors were not significantly different between groups. No serious adverse event was observed.

Conclusion: Our finding did not show the superiority of fixed-schedule ER-MTP over placebo when added to an intensive biweekly outpatient psychosocial treatment. Further studies using individually tailored flexible-dose regimes might provide new insights regarding the safety and efficacy of psychostimulant maintenance treatment for MA dependence.

Keywords: Methamphetamine, Amphetamine-Related disorders, Treatment, Methylphenidate

Conflicts of Interest: None declared

Funding: Tehran University of Medical Sciences

\section{*This work has been published under CC BY-NC-SA 1.0 license.} Copyright $\odot$ Iran University of Medical Sciences

Cite this article as: Noroozi A, Motevalian SA, Zarrindast MR, Alaghband-Rad J, Akhondzadeh Sh. Adding extended-release methylphenidate to psychological intervention for treatment of methamphetamine dependence: A double-blind randomized controlled trial. Med J Islam Repub Iran. 2020 (14 Oct);34:137. https://doi.org/10.47176/mjiri.34.137

\section{Corresponding author: Dr Shahin Akhodzadeh, s.akhond@neda.net}

Department of Neuroscience and Addiction Studies, School of Advanced Technologies in Medicine (SATiM), Tehran University of Medical Sciences, Tehran, Iran

2. Iranian National Center for Addiction Studies (INCAS), Tehran University of Medical Sciences, Tehran, Iran

3. Department of Epidemiology, School of Public Health, Iran University of Medical Sciences, Tehran, Iran

4. Cognitive and Neuroscience Research Center (CNRC), Tehran Medical Sciences Branch, Islamic Azad University, Tehran, Iran

5. Medical Genomics Research Center, Tehran Medical Sciences Branch, Islamic Azad University, Tehran, Iran

6. School of Cognitive Sciences, Institute for Research in Fundamental Sciences (IPM), Tehran, Iran

7. Department of Pharmacology, School of Medicine, Tehran University of Medical Sciences, Tehran, Iran

8. Department of Psychiatry, Roozbeh Hospital, Tehran University of Medical Sciences, Tehran, Iran

9. Psychiatric Research Center, Roozbeh Hospital, Tehran University of Medical Sciences, Tehran, Iran $\uparrow$ What is "already known" in this topic:

No effective pharmacotherapy has been found for stimulant dependence yet. Preclinical studies support the potential efficacy of agonist-like maintenance treatment with methylphenidate (MTP) for methamphetamine (MA) dependence. Previous controlled clinical studies suggest positive trends for MTP in MA treatment.

\section{$\rightarrow$ What this article adds:}

Adding-on a fixed-dose extended-release methylphenidate treatment regime to intensive psychological treatments was not superior to placebo in treatment retention, weekly MA negative urine test, and craving outcomes. Further studies to explore the effect of dose and treatment regime on treatment efficacy are needed. 


\section{Introduction}

Annual prevalence of last year amphetamines use estimated to be $0.7 \%$ in 2016 globally (1). For centuries, opioids have been the main drug of use in Iran drug scene (2), although a methamphetamine (MA) use epidemic has emerged in the country during the last decade (3). MA use rapidly spread in the country as a result of positive outcome expectations and low perceived risk (4) and treatment demand for MA treatment was increased (3).

Among illicit drugs, amphetamines are the second leading factor attributable to the burden of disease after opioids at the global level (5). MA use is associated with an increased risk for cardiovascular diseases, psychiatric disorders, injecting, and sexual-related high-risk behaviors and mortality (3, 6-8). MA use is associated with an increased risk for psychosis, anxiety, depression, suicidal ideation, suicidal attempts, and problems with controlling anger and violence (6-7).

No pharmacological treatment has been introduced for MA use disorder (9), and psychosocial treatments are the only identified effective interventions $(10,11)$. Using agonist maintenance treatments has been successful for the treatment of opioids $(12,13)$ and tobacco use disorders (14). Evidence regarding the efficacy of agonist treatments for stimulant (MA) use disorder is limited (15).

It has been shown that stimulants' use is associated with brain dopaminergic system deficits (16), as a result of reactive species formation (17). However, using psychostimulants has a dose-dependent impact on extracellular dopamine concentrations (18). There is a distinct pattern of postsynaptic dopamine receptors' activation and potential neurotoxic effects among those use stimulants for therapeutic purposes compared to people who use high doses often seen in stimulant addiction (18-21).

Pre-clinical studies support methylphenidate (MTP) as a potential candidate for the agonist-like treatment of stimulant dependence $(15,22)$. MTP is a psychostimulant medication, which has been approved for the treatment of attention deficit hyperactivity disorder (ADHD) among children, adolescents (23) and adults (24). A Finish study showed the superiority of slow-release MTP (SR-MTP) $(54 \mathrm{mg} /$ day) over placebo on MA abstinence outcomes among people with amphetamine or MA dependence that use drugs through injection (25). A more recent study in Finland and New Zealand did not find any differences between extended-release MTP (ER-MTP) (54mg/day) and placebo on treatment retention and MA abstinence outcomes, although ER-MTP treatment was associated with higher retention rate as compared to placebo from week 6 to 22 of the study (26).

As a result of preliminary positive trends for MTP, the aim of this randomized controlled trial (RCT) is to evaluate the efficacy of extended-release MPH (ER-MTP) compared to placebo for MA dependence.

\section{Methods}

\section{Design}

The study was a 12 -week phase II, parallel groups, randomized, placebo-controlled trial of ER-MTP added to standard psychological treatment of MA dependence.

\section{Participants}

Inclusion criteria were age 18 to 65 years old, diagnosis of MA dependence according to DSM-IV-TR (27), positive rapid urine test for MA, and voluntary informed written consent for the treatment. Exclusion criteria were current diagnosis of other substance use disorders (except nicotine), current or past history of other axis I psychiatric disorders (except depression), having a significant risk of suicide or violence to others, significant neurologic or medical diseases, pregnancy and lactation, history of childhood ADHD, development of psychotic symptoms required pharmacotherapy and IQ lower than 70. Eligibility of the study participants was determined through an interview conducted by the study physician.

\section{Recruitment and Consent}

Participants were recruited from treatment-seeking patients referring to Mehrayeen outpatient specialized drug treatment center in Tehran from September 2013 to June 2016. The recruitment methods included the announcement of the study among medical doctors of other drug treatment centers and asked them to refer potential participants to the study. Patients who referred to the study site and sought MA dependence treatment were screened for eligibility criteria.

\section{Allocation, Concealment, and Blinding}

Participants $(\mathrm{n}=62)$ randomized to receive either ERMTP $(n=32)$ or placebo $(n=30)$ using random sequence blocks with a block size of 4 generated by Microsoft Excel software. Concealment of allocation was performed using sequentially numbered, opaque, and sealed envelopes. Treatment providers of the drug treatment center were all blinded to the treatment group throughout the study.

\section{Interventions}

Study participants received either ER-MTP or placebo through the same size, color and shape gel capsules filled with ER-MTP (20mg; Stimdate ${ }^{\circledR}$, manufactured by Mehrdarou Pharmaceutical Company) in the active arm or starch in the placebo arm. MPH dose was ER-MPH $20 \mathrm{mg}$ (once a day at morning) for week $1,40 \mathrm{mg}$ (20mg twice a day at morning and noon) for week 2 and $60 \mathrm{mg}$ (20mg thrice a day at morning, noon and afternoon) for weeks 3 to 12. All participants were visited twice weekly by the study physician in the morning. At visit days, participants were requested to take their morning doses under the direct supervision of the dispensing nurse. Study participants were allowed to receive other doses as take-home in childproof pill containers. Study participants received medication-adherence and safety psychoeducation by the treatment team including study physician and clinical psychologist. They were informed that their medication is scheduled and diverting their doses results to exclusion from the trial.

All participants also received individual treatment sessions based on the modified Matrix model twice a week by 
a trained clinical psychologist. Modified Matrix treatment for stimulant treatment is a structured treatment consisted of 24 sessions using motivational enhancement, psychoeducational and cognitive, behavioral treatment techniques (28).

All treatment interventions and assessments were provided free of charge. Potential benefits and adverse effects of study participation were clearly explained for participants, and informed written consent was obtained. It was explained to participants that they could exit from the study whenever they want and this would not have any negative effect on receiving routine services from the study site. The study protocol and questionnaires were approved by the Ethics Committee of Vice-Chancellor for Research, Tehran University of Medical Sciences (TUMS) (Code: 16507). The study was registered in Iranian Registry of Clinical Trials (registration code: IRCT201202281556N38)

\section{Measures}

A physical exam was carried out by study physician at baseline. Urine sample was tested for methamphetamine (manufactured by ACON, cut-off point for minimal sensitivity of $500 \mathrm{ng} / \mathrm{ml}$ ) on a weekly basis. A pregnancy test was conducted for female participants at baseline. Eligible participants were randomized to receive either ER-MTP or placebo after providing informed written consent.

A comprehensive history of drug and alcohol use and severity of problems in medical, psychiatric, employment and family domains were measured by Addiction Severity Index (ASI)-5th Edition at baseline and week 12 (29). Farsi version of ASI-5th Edition has shown good reliability and validity (30). ASI composite score (CSs) for each functional domain was calculated using the ASI manual; with higher scores represent higher addiction severity. Methamphetamine withdrawal was measured using Amphetamine Withdrawal Questionnaire (AWQ) (31) and Amphetamine Selective Severity Assessment (ASSA) (32) at baseline and day 3-4, week 1 , week 4 and week 12 . AWQ is a 10 -item questionnaire measuring MA withdrawal severity over the previous 24 hours. Each item scored on a four-point Likerttype scale, from 0 (not at all) to 4 (very much). ASSA is an 18-item questionnaire which used to provide information on a broader range of MA withdrawal. ASSA items score from 0 to 7 , with higher scores indicated greater severity. A preliminary analysis of our data showed that Cronbach's alpha coefficients for AWQ and ASSA were excellent (91\%) and good (84\%), respectively. The highest degree of craving during the last 24 hours was measured with a visual analogue scale (VAS) on a weekly basis (33). VAS is a single-item assessment in which participants were asked to rate their highest level of MA craving during the last 24 hours on a $10 \mathrm{~cm}$ ruler graded from 0 to 100 (33-34). We used beck depression inventory- second edition (BDI-II) to measure depression (35). The Farsi version of BDI-II has shown acceptable reliability and validity (36). To measure injecting and sexual-related high-risk behaviors, we used standard Iranian bio-behavioral survey questionnaires for people who use drugs (37-39) at baseline, weeks 4 and 12. Study questionnaires were completed by a trained master level clinical psychologist. Blood pressure of participants and adverse effects were monitored on a weekly basis by the study physician.

\section{Outcomes}

Primary outcomes of the study were percent of the weekly negative urine tests for MA measured by a rapid urine test, addiction severity and craving. The secondary outcome measures were treatment retention, self-report of MA use, self-report of using other drugs, methamphetamine withdrawal, depression, high-risk behaviors and adverse events.

\section{Sample size}

Assuming a clinically significant between-group differences of $15 \%$ on a weekly negative urine test, a standard deviation of $20 \%$, a power of $80 \%$ and a two-sided significance level of 0.05 , a minimal sample size of 58 was calculated. Considering the high attrition rate, we increased the planned sample size to 70 .

\section{Data analysis}

SPSS software version 22 (IBM Corp, Armonk, NY) was used for data analysis. We used intention to treat analysis to compare treatment outcomes between groups. Continuous variables are presented as mean \pm standard deviation, and categorical variables are presented as number and percentage. To compare groups in terms of treatment retention, and urine test results for $\mathrm{MA}$, analysis of variance (ANOVA), generalized linear model (GLM) was used. Generalized estimating equation (GEE) was used for between-groups comparison of craving. ANOVA was used to compare groups differences in terms of depression, withdrawal and high-risk behaviors outcomes.

\section{Results}

Among 161 of clients referred to the study site, 62 met eligibility criteria. The eligible participants were randomized to ER-MTP $(n=32)$ and placebo $(n=30)$. Among 62 study participants who received at least one dose of study medication, $31(50 \%)$ completed 12 weeks of study. The retention rate for ER-MTP and placebo groups were $53.13 \%$ and $46.67 \%$, respectively. Flow diagram of the study was presented in Figure 1.

\section{Demographic characteristic}

The average age of participants in intervention and control groups was $31.9( \pm 2.77)$ and $32.33( \pm 3.48)$ years, respectively. Female participants in intervention and control groups were $28.1 \%$ and $30 \%$, respectively. Baseline demographic characteristics of participants in control and intervention groups did not show significant differences at baseline assessment (Table 1).

\section{History of drug use}

History of MA use, use of other drugs, severity of depression, CSs of ASI domains including medical, employment, alcohol, drug, family, legal and psychiatric domains did not have significant differences between groups at baseline assessment (Table 1). 


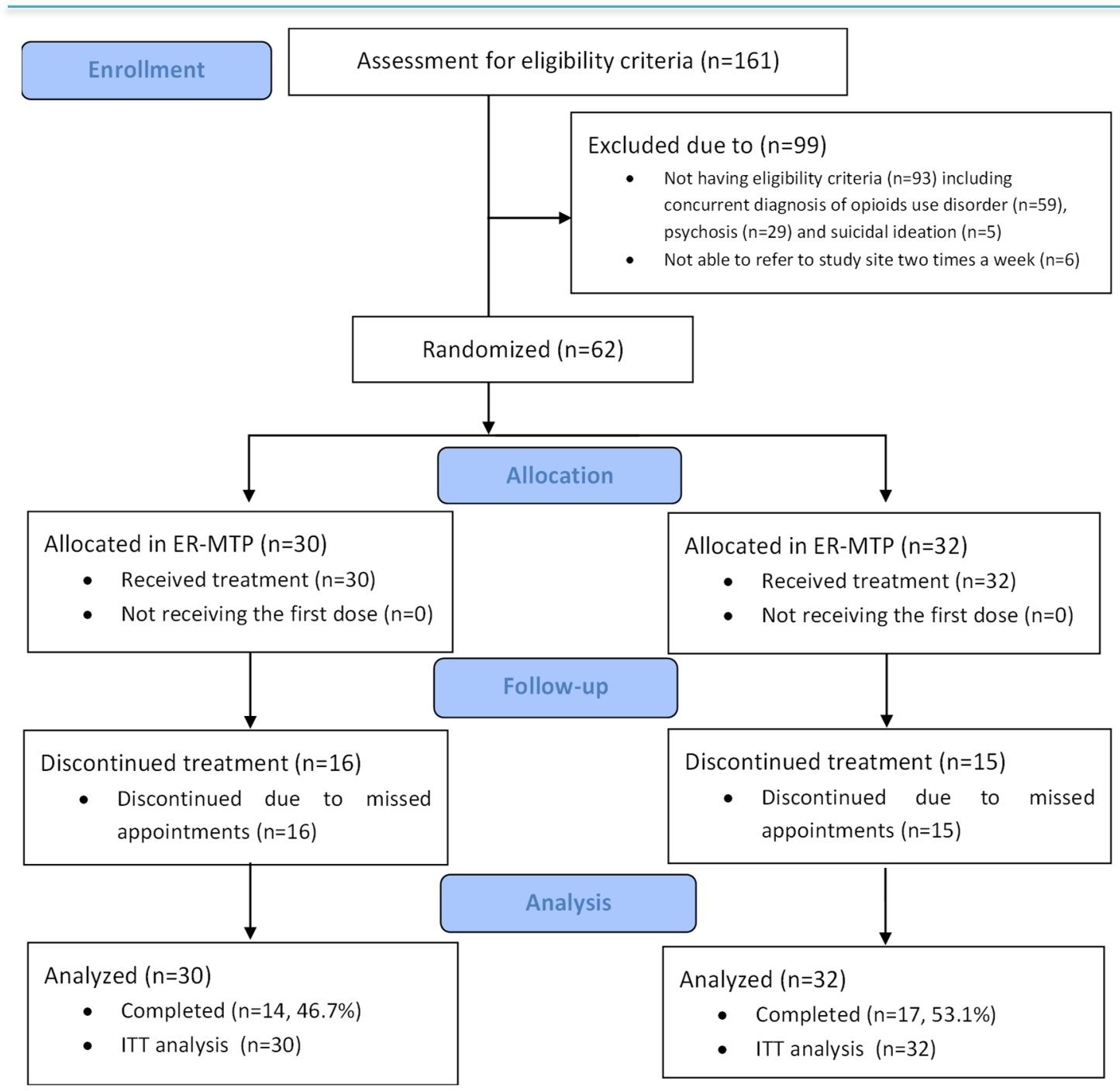

Fig. 1. CONSORT diagram

\section{Treatment retention}

Treatment retention in ER-MTP and placebo groups was $53.1 \%$ and $46.7 \%$, respectively. Retention rate as a categorical variable compared between groups using GLM analysis and did not show significant differences between groups $(\mathrm{p}=0.610)$ (Fig. 2).

\section{MA abstinence}

Percent of positive MA urine tests did not show significant differences between study groups during the 12 weeks of the study, using GLM analysis ( $p=0.766$ ) (Fig. 3). Terminal abstinence defined as the percentage of negative MA urine tests between groups at week 12. There was a trend toward better abstinence rate in ER-MTP (82.4\%) compared to placebo $(78.6 \%)$, although the difference between groups was not significant $(\mathrm{p}=0.215)$. The average number of MA use days during the last month in the ER-MTP group (1.35 \pm 1.41$)$ was not significantly different from the placebo group (1.29 \pm 1.14$)(\mathrm{p}=0.887)$. Differences between the reduction in the average number of MA use days during the last month in the ER-MTP group $(27.94 \pm 2.49)$ were not significant with the placebo group $(28.29 \pm 1.90)(\mathrm{p}=0.673)$.

\section{Abstinence of other drugs}

Changes in self-report of the number of days using drug and alcohol during last month at baseline and week 12 were not significantly different between groups (Table 2).

Craving: Highest level of craving during the last 24 hours was not significantly different between groups at baseline (ER-MTP: 44.38 \pm 10.6 , placebo: $44.67 \pm 9.6 \mathrm{p}=0.887$ ). Mean changes in craving scores during the course of study did not show a significant difference between groups using $\operatorname{GEE}(\mathrm{p}=0.525)$.

\section{Withdrawal}

Withdrawal scores measured by AWQ (ER-MTP: 5.7 \pm 3.3 placebo: $5.944 \pm 29 \mathrm{p}=0.682$ ) and ASSA (ER-MTP: $19.16 \pm 8.3$, placebo: $19.80 \pm 9.6 \mathrm{p}=0.783$ ) did not have a significant difference at baseline. Methamphetamine withdrawal measured by AWQ and ASSA decreased significantly in both groups during the study; however, betweengroup comparisons did not find significant differences in changes in AWQ $(\mathrm{F}=0.533 \mathrm{p}=0.225)$ and ASSA $(\mathrm{F}=0.425$ 
Table 1. Demographic characteristics of participants, history of drug use, CSs of ASI domains and depression at baseline

\begin{tabular}{|c|c|c|c|}
\hline & \multicolumn{2}{|c|}{ Study group } & \multirow[t]{2}{*}{$\mathrm{p}$} \\
\hline & ER-MTP & Placebo & \\
\hline \multicolumn{4}{|l|}{ Demographic chracteristics } \\
\hline Age & $31.90( \pm 2.77)$ & $32.33( \pm 3.48)$ & 0.645 \\
\hline \multicolumn{4}{|l|}{ Gender } \\
\hline • Male & $71.9 \%$ & $70.0 \%$ & 0.574 \\
\hline - Female & $28.1 \%$ & $30.0 \%$ & \\
\hline \multicolumn{4}{|l|}{ Marital status } \\
\hline$\bullet \quad$ Married & $62.5 \%$ & $49.3 \%$ & 0.234 \\
\hline - $\quad$ Divorced & $21.9 \%$ & $20.0 \%$ & \\
\hline - $\quad$ Never married (signle) & $15.6 \%$ & $30.7 \%$ & \\
\hline Completed years of eduction & $11.8( \pm 1.6)$ & $11.4( \pm 2.3)$ & 0.735 \\
\hline \multicolumn{4}{|l|}{ Occupational status } \\
\hline • Full-time & $59.4 \%$ & $46.7 \%$ & 0.343 \\
\hline - $\quad$ Part-time & $21.8 \%$ & $23.3 \%$ & \\
\hline • Unemployed & $18.8 \%$ & $30 \%$ & \\
\hline \multicolumn{4}{|l|}{ Living status (with whom) } \\
\hline - With family & $90.6 \%$ & $96.7 \%$ & 0.491 \\
\hline - With friends & $3.1 \%$ & $0.0 \%$ & \\
\hline - Alone & $6.3 \%$ & $3.3 \%$ & \\
\hline \multicolumn{4}{|l|}{ Place of residence owned by participants or their families } \\
\hline - Yes & $34.4 \%$ & $30.0 \%$ & 0.734 \\
\hline - $\quad$ No & $65.6 \%$ & $70.0 \%$ & \\
\hline \multicolumn{4}{|l|}{ Having valid car license } \\
\hline$\bullet \quad$ Yes & $78.1 \%$ & $86.7 \%$ & 0.125 \\
\hline - $\quad$ No & $21.9 \%$ & $13.3 \%$ & \\
\hline \multicolumn{4}{|l|}{ Drug Use History } \\
\hline Years of regular methamphetamine use & $5.7( \pm 1.3)$ & $5.1( \pm 1.8)$ & 0.712 \\
\hline Days of methamphtamine use during last month & $29.6( \pm 1.5)$ & $29.8( \pm 1.1)$ & 0.836 \\
\hline Year of regular opium use & $1.5( \pm 1.5)$ & $1.2( \pm 1.2)$ & 0.431 \\
\hline Days of opium use during last month & $3.1( \pm 3.9)$ & $2.1( \pm 3.1)$ & 0.532 \\
\hline Year of regular heroin use & $0.7( \pm 1.1)$ & $0.9( \pm 1.3)$ & 0.281 \\
\hline Days of heroin use during last month & 0 & 0 & 1 \\
\hline Years of regular alcohol use & $0.1( \pm 0.3)$ & $0.1( \pm 0.3)$ & 0.821 \\
\hline Days of alcohol use during last month & $1.1( \pm 2.2)$ & $1.2( \pm 1.8)$ & 0.782 \\
\hline Days of alcohol use at intoxication level during last month & $0.7( \pm 1.3)$ & $0.6( \pm 1.2)$ & 0.751 \\
\hline Years of regular sedatives use & $0.3( \pm 0.5)$ & $0.3( \pm 0.5)$ & 0.842 \\
\hline Days of sedative use during last month & $4.1( \pm 3.9)$ & $3.1( \pm 3.8)$ & 0.421 \\
\hline Years of regular cannabis use & $1.1( \pm 1.1)$ & $1.3( \pm 1.2)$ & 0.421 \\
\hline Days of cannabis use during last month & $2.9( \pm 3.5)$ & $4( \pm 5.1)$ & 0.195 \\
\hline Years of polysubstance use & $0.9( \pm 0.8)$ & $1.0( \pm 1.0)$ & 0.743 \\
\hline Days of polysubstance use last month & $8.5( \pm 4.5)$ & $8.3( \pm 5.9)$ & 0.839 \\
\hline \multicolumn{4}{|l|}{ Addiction severity composite scores } \\
\hline Medical & $0.14( \pm 0.12)$ & $0.13( \pm 0.98)$ & 0.775 \\
\hline Employment & $0.37( \pm 0.25)$ & $0.39( \pm 0.27)$ & 0.634 \\
\hline Alcohol & $0.05( \pm 0.09)$ & $0.06( \pm 0.10)$ & 0.491 \\
\hline Drug & $0.55( \pm 0.02)$ & $0.54( \pm 0.03)$ & 0.743 \\
\hline Legal & $0.04( \pm 0.17)$ & $0.11( \pm 0.25)$ & 0.161 \\
\hline Familial & $0.48( \pm 0.10)$ & $0.52( \pm 0.14)$ & 0.576 \\
\hline Psychiatric & $0.39( \pm 0.13)$ & $0.40( \pm 0.15)$ & 0.761 \\
\hline Beck depression inventory score & $18.3( \pm 8.5)$ & $18.4( \pm 8.3)$ & 0.656 \\
\hline
\end{tabular}

$\mathrm{p}=0.325)$ scores.

\section{ASI CSS}

Both groups showed a significant decrease in ASI CSs for medical, drug, family and psychiatric domains at week 12 as compared to baseline, although the differences between groups were not significant. CSs for alcohol and legal domains were very low for both groups at baseline and did not show a significant decrease at week 12 . The change in CSs at baseline and 12 weeks follow-up was not significantly different between ER-MTP and placebo groups (Table 2).

\section{Depression}

Severity of depression was measured by BDI-II in the
ER-MTP group was $18.3( \pm 8.5)$ at baseline which was decreased to $13.4( \pm 4.7)$ and $9.6( \pm 2.4)$, respectively. BDI-II score in the placebo group was $18.4( \pm 8.3)$ at baseline which was decreased to $12.2( \pm 5.1)$ and $9.7( \pm 1.9)$ at weeks 4 and 12, respectively. Depression scores at week 12 both in ER-MTP and placebo groups, were significantly lower than baseline $(\mathrm{p}=0.001)$. The change in depression scores in the course of study was not significantly different between groups $(\mathrm{F}=0.376, \mathrm{p}=0.544)$.

\section{High-risk behaviors}

HIV-related high-risk behaviors questionnaire consisted 
Efficacy of methylphenidate treatment of methamphetamine dependence

Table 2. Self-report of using other drugs and CSs of ASI at baseline and week 12

\begin{tabular}{|c|c|c|c|c|c|}
\hline & \multicolumn{2}{|c|}{ Baseline } & \multicolumn{2}{|c|}{ Week 12} & \multirow[t]{2}{*}{$\mathrm{p}$} \\
\hline & ER-MTP & Placebo & ER-MTP & Placebo & \\
\hline \multicolumn{6}{|l|}{ Drug use history } \\
\hline Days of opium use during last month & $3.1( \pm 3.9)$ & $2.1( \pm 3.1)$ & $0.1( \pm 0.2)$ & $0.1( \pm 0.5)$ & 0.566 \\
\hline Days of heroin use during last month & 0 & 0 & 0 & 0 & 1 \\
\hline Days of alcohol use during last month & $1.1( \pm 2.2)$ & $1.2( \pm 1.8)$ & $0.6( \pm 0.8)$ & $0.6( \pm 0.9)$ & 0.955 \\
\hline Days of alcohol use at intoxication level during last month & $0.7( \pm 1.3)$ & $0.6( \pm 1.2)$ & $0.3( \pm 0.6)$ & $0.2( \pm 0.6)$ & 0.708 \\
\hline Days of sedative medications use during last month & $4.1( \pm 3.9)$ & $3.1( \pm 3.8)$ & $1.2( \pm 1.7)$ & $1.0( \pm 1.5)$ & 0.759 \\
\hline Days of cannabis use during last month & $2.9( \pm 3.5)$ & $4( \pm 5.1)$ & $2.2( \pm 3.2)$ & $2.5( \pm 6.3)$ & 0.854 \\
\hline Days of polysubstance use & $8.5( \pm 4.5)$ & $8.3( \pm 5.9)$ & $7.9( \pm 4.8)$ & $7.3( \pm 4.9)$ & 0.981 \\
\hline \multicolumn{6}{|l|}{ ASI Composite Scores (CSs) } \\
\hline Medical & $0.14( \pm 0.12)$ & $0.13( \pm 0.98)$ & $0.07( \pm 0.08)$ & $0.08( \pm 0.10)$ & 0.425 \\
\hline Employment & $0.37( \pm 0.25)$ & $0.39( \pm 0.27)$ & $0.34( \pm 0.18)$ & $0.37( \pm 0.24)$ & 0.812 \\
\hline Alcohol & $0.05( \pm 0.09)$ & $0.06( \pm 0.10)$ & $0.04( \pm 0.01)$ & $0.05( \pm 0.07)$ & 0.781 \\
\hline Drug & $0.55( \pm 0.02)$ & $0.54( \pm 0.03)$ & $0.15( \pm 0.03)$ & $0.14( \pm 0.04)$ & 0.433 \\
\hline Legal & $0.04( \pm 0.17)$ & $0.11( \pm 0.25)$ & $0.04( \pm 0.08)$ & $0.09( \pm 0.08)$ & 0.278 \\
\hline Familial & $0.48( \pm 0.10)$ & $0.52( \pm 0.14)$ & $0.08( \pm 0.06)$ & $0.15( \pm 0.08)$ & 0.202 \\
\hline Psychiatric & $0.39( \pm 0.13)$ & $0.40( \pm 0.15)$ & $0.10( \pm 0.07)$ & $0.15( \pm 0.07)$ & 0.336 \\
\hline
\end{tabular}

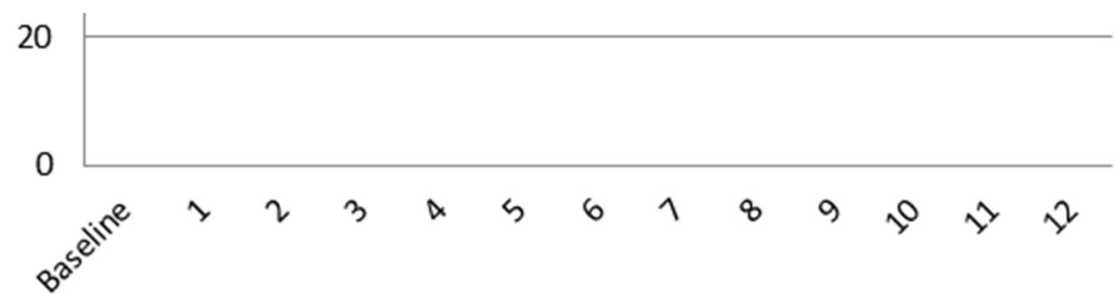

Fig. 2. Retention rate by study groups

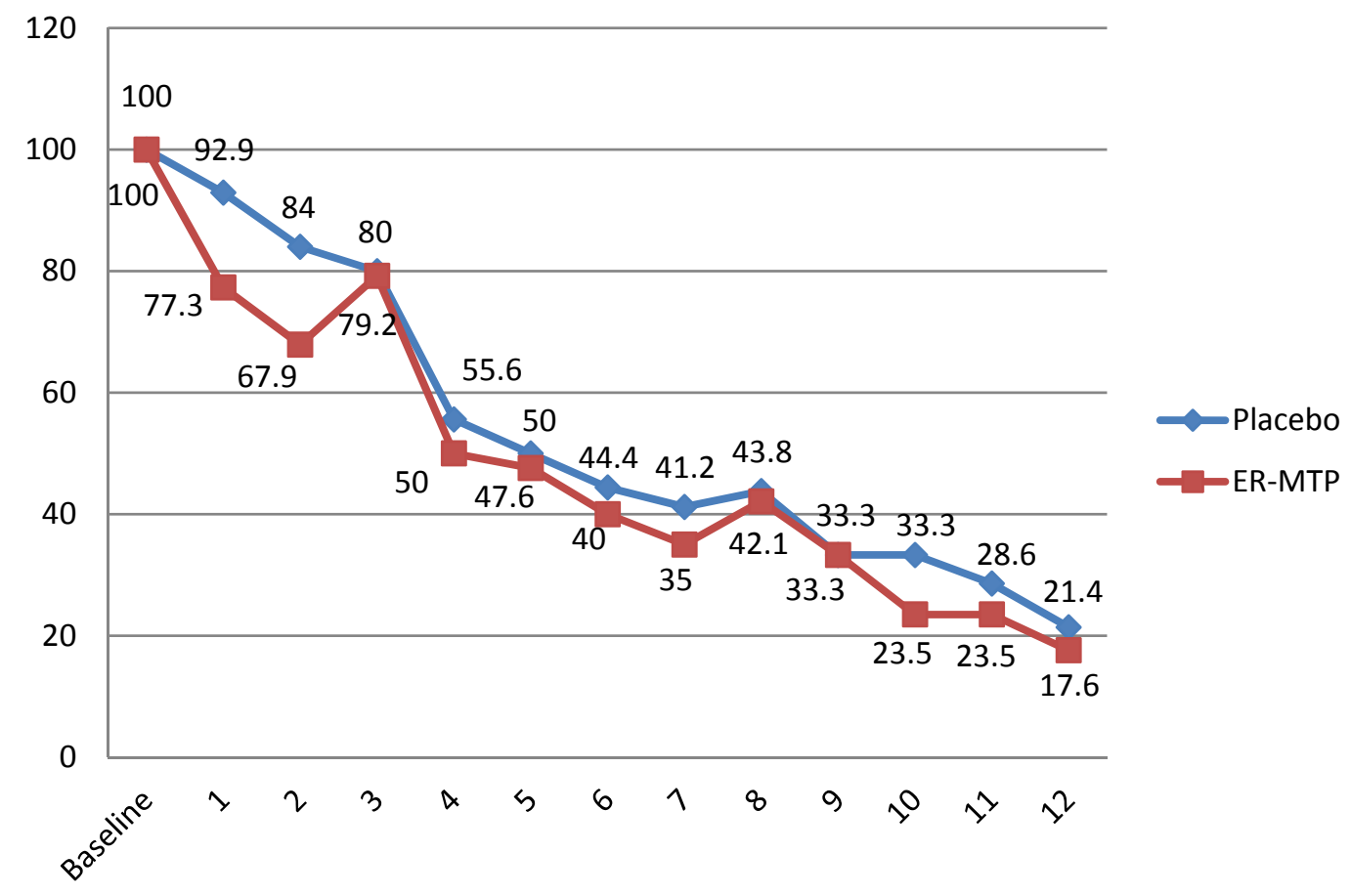

Fig. 3. Percent of positive weekly urine tests

of items measuring injecting and unprotected sexual intercourse during last month. The participants did not accept any history of injection during last month at baseline and weeks 4 and 12 follow-ups. The average number of sexual intercourses during last month in ER-MTP at baseline assessment was $6.9( \pm 5.6)$ which decreased to $5.7( \pm 4.0)$ and $4.1( \pm 2.9)$ at weeks 4 and 12 , respectively. The average number of sexual intercourses was significantly decreased in the ER-MTP group in week 12 as compared to baseline $(p<0.001)$. The average number of sexual intercourses in the placebo group at baseline was $7.0( \pm 6.1)$ and decreased to $4.7( \pm 3.9)$ and $3.8( \pm 2.9)$ at 4 and 12 weeks follow-up, respectively. Reduction in the average number of sexual intercourses as compared to baseline assessment significantly decrease $(\mathrm{p}=0.000)$. The comparison of groups in terms of the average number of sexual intercourses during the study was not significantly different $(\mathrm{p}=0.753)$.

\footnotetext{
6 http://mjiri.iums.ac.ir

Med J Islam Repub Iran. 2020 (14 Oct); 34:137.
} 
The average number of sexual intercourse without a condom during last months at baseline was $2.9( \pm 2.1)$ which decreased to $2.3( \pm 1.4)$ and $1.5( \pm 1.0)$ at week 4 and 12 follow-ups and at week 12 of the follow up was significantly lower than the baseline $(\mathrm{p}<0.001)$. The variable in the placebo group at baseline was $3.6( \pm 2.1)$ and decreased to 1.8 $( \pm 1.3)$ and $1.4( \pm 0.9)$ at weeks 4 and 12 follow-ups and the decrease as compared to baseline was significant $(p=0.000)$. Although both groups showed a significant decrease in the number of sexual intercourse during the last month without condom, between groups comparison did not reveal a significant difference during 12 weeks study $(\mathrm{p}=0.533)$.

Percent of unprotected sex during last month in the ERMTP group at baseline was $44.5 \%$ which was decreased to $36.7 \%$ and $24.5 \%$ at weeks 4 and 12 follow-ups, respectively and the decrease in the variable at week 12 as compared to baseline was significant $(p=0.001)$. Similarly, percent of unprotected sex during last month in the placebo group was decreased from $47.3 \%$ at baseline to $25.5 \%$ and $25 \%$ at weeks 4 and 12, respectively and the decrease in the variable from baseline to week 12 was significant $(p=0.001)$. Although both groups showed a significant decrease in the percent of unprotected sex during the last month, between groups comparison, did not find a significant difference between groups $(p=0.225)$.

Percent of participants who reported not using condoms in their last sex in the ER-MTP group was $68.8 \%$ which was decreased to $54.5 \%$ and $29.4 \%$ at weeks 4 and 12 follow-ups, respectively, and decrease in the variable at weeks 12 as compared to baseline was significant $(p=0.001)$. In placebo group also percent of participants who reported not using a condom in their last sex was decreased from 56.7\% at baseline to $33.3 \%$ and $28.6 \%$ at weeks 4 and 12 followups, respectively and the change in the variable at week 12 was significant as compared to baseline $(p=0.001)$. The change in percent of participants who report not using a condom in their last sexual intercourse was not significantly different between study groups $(\mathrm{p}=0.345)$.

\section{Side effects}

No serious side effects, including suicide attempt, aggression, psychosis or chest pain was not seen. The monitoring of blood pressure did not show an increase in blood pressure above the normal range in any participant. There was no report of an overt attempt of study participants to sell study medication in clinic premises; therefore, no participant was excluded from the study due to diversion of study medication. A range of side effects including insomnia, headache, dizziness, anxiety, dysphoria, decreased appetite, muscle aches, nausea and irritability were observed among study participants, but all were mild and did not necessitate discontinuation of study medication.

\section{Post-hoc analysis}

Figure 3 shows that there was a separation between ERMTP and placebo groups with regard to percent of weekly MA positive urine tests in week 1 and 2. Although the change in percent of weekly MA urine tests did not show significant between-groups differences over the course of study, a post-hoc analysis showed that a decrease in the percent of MA positive urine test during the first 2 weeks of study in ER-MTP was significantly higher than the placebo group $(p=0.019)$. ER-MTP group also showed significantly lower craving scores during the first 2 weeks of the study $(p=0.001)$. Significantly lower severity of withdrawal measured by AWQ $(p=0.024)$ and ASSA $(p=0.020)$ in the first week of the study (baseline, day 3 and week 1 assessments) was evident in ER-MTP group as compared to placebo.

\section{Discussion}

Study results did not find the superiority of ER-MTP $(60 \mathrm{mg} /$ day) over placebo in percent of negative weekly urine tests, terminal abstinence and self-report number of MA use during last month during 12 weeks of study, although both groups showed a significant decrease in mentioned outcomes as compared to baseline. Although the percent of participants who completed the study was higher in the ER-MTP group as compared to placebo but the difference was not statistically significant.

These results were consistent with findings of an RCT comparing slow-release dexamphetamine $(110 \mathrm{mg} /$ day $)$ with placebo during 12 weeks in Australia (40), in which both groups showed a significant decrease in MA use, although between groups comparisons could not find significant differences. A 12-weeks open-label comparison of dexamphetamine treatment $(60 \mathrm{mg} /$ day $)$ with placebo also did not show a significant difference between groups in terms of weekly negative urine tests, the cost paid for MA and HIV-related high-risk behaviors (41). An 8-weeks RCT in San Fransisco, US on the efficacy of slow-release dexamphetamine (30mg twice a day) also did not show significant between-groups differences in treatment retention, self-reported MA use and negative urine test for MA (42). Two RCTs comparing the efficacy of osmotic-release oral system MTP (54mg/day) with placebo among people who inject MA/amphetamines (25) and people with MA dependence (43), reported significantly lower MA positive urine test in ER-MTP group compared to placebo, although two other RCTs could not replicate these results $(26,44)$.

Inconsistent outcomes on MA negative urine between studies using psychostimulant maintenance treatment approaches might be the result of different medication adherence across studies. Further studies to measure the effect of medication adherence on treatment outcomes are warranted.

Our finding showed no significant differences in treatment retention between the study groups, which was consistent with the results of previous studies using dopamine agonist maintenance treatment including slow-release dexamphetamine (30 $\mathrm{mg}$ twice a week) (42) and ER-MTP $(54 \mathrm{mg}$ /day) $(25,26,43,44)$, although one RCT reported higher retention rate in slow-release dexamphetamine group (individually tailored up to $110 \mathrm{mg}$ /day) as compared to placebo (40). This suggests the importance of investigating the safety and efficacy of individualized regimes of dopamine maintenance treatments for MA use. One RCT showed a significantly higher retention rate in ER-MTP ( $54 \mathrm{mg} /$ day) group compared to the placebo group from 
week 6 to week 22 (26). Further studies with higher sample sizes and longer follow-up are needed to investigate long term treatment efficacy of ER-MTP.

In our study, the decrease in weekly craving scores throughout of study was not significantly different between groups which was consistent with two ER-MTP vs. placebo RCTS for 22-weeks (26) and 14 weeks (44), respectively. However, it was inconsistent with the result of another RCT for 10 -weeks which only measured carving at three time points including baseline, week 6 and 10 (43). Our study and all other mentioned RCTs used a fixed schedule dose regime of ER-MTP. Experimental studies have shown higher serum levels of methadone $(45,46)$ or buprenorphine (47) are associated with lower craving levels among people with opioid use disorder. A finding which supported with clinical studies showing higher methadone $(48,49)$ and buprenorphine doses $(12,50)$ are associated with more illicit opioid use abstinence in opioid agonist maintenance programs. Further studies are needed to investigate the effect of different doses and treatment regimens on MA craving.

Change in withdrawal severity scores over the course of study was not significantly different between groups, although the post-hoc analysis of our results showed that during the first week of the study, participants in the ER-MTP group reported significantly lower levels of withdrawal severity as compared to placebo. These findings were consistent with the results of an RCT comparing the efficacy of an individualized dose regime of slow-release dexamphetamine (up to $110 \mathrm{mg}$ per day) among people with MA dependence (40). Likewise, participants in the active treatment group showed significantly more reduction in withdrawal scores at the end of the stabilization phase (first 14 days) compared to the placebo. A trend toward more reduction in withdrawal was seen in the slow-release dexamphetamine group, although it was not significant over the course of study.

The post-hoc analysis also showed a significantly more reduction in the percent of MA positive urine test and craving during the first 2 weeks of study in the ER-MTP group as compared to the placebo. A study on the natural course of withdrawal and craving among people with MA dependence indicated that acute phase of MA withdrawal lasts for 7-10 days after MA cessation, although MA craving levels will remain high for weeks and months (51). Our findings suggest that the potentially positive effect of ER-MTP might be mediated through alleviating withdrawal severity during the first 2 weeks of treatment, but its effect has been disappeared after passing of the high initial peak of withdrawal. A similar pattern has been reported with low, fixeddose opioid agonist maintenance treatments, which could effectively manage withdrawal signs and symptoms and increase short-term abstinence but could not significantly improve long-term abstinence over placebo (12).

We did not see any serious side effects in study groups, a finding which was consistent with previous studies using ER-MTP $(25,44)$ or other psychostimulants $(40,42)$ for MA treatment.

Limitations of this study include a small sample size that limits subgroup analysis based on gender or addiction severity and high attrition rate. Treatment retention was not significantly different between groups; it suggests that reasons for attrition are not related to study medication.

\section{Conclusion}

Our findings showed that fixed-schedule ER-MTP treatment for MA dependence was safe. Although ER-MTP treatment was more effective than placebo on MA negative urine test, withdrawal and craving during weeks 1 and 2; it did not significantly improve treatment retention, abstinence, craving and withdrawal during the 12-week course of the study. Further studies using individualized, flexibledose regimes might provide new insights regarding the effect of psychostimulant maintenance treatment for MA dependence.

\section{Acknowledgment}

The authors would like to acknowledge study participants, their families and treatment staff who collaborated in this research.

\section{Conflict of Interests}

The authors declare that they have no competing interests.

\section{References}

1. United Nation Office on Drug and Crime (UNODC). World Drug Report 2018. United Nations publication, Sales No. E.18.XI.9, 2018.

2. Momtazi S, Noroozi A, Rawson R. An Overview of Iran Drug Treatment and Harm Reduction Programs. In: el-Guebaly N, Carrà G, Galanter M. (Eds) Textbook of Addiction Treatment: International Perspectives. Springer, Milano, 2015.

3. Shadloo B, Amin-Esmaeili M, Haft-Baradaran M, Noroozi A, Ghorban-Jahromi R, Rahimi-Movaghar A. Use of amphetamine-type stimulants in the Islamic Republic of Iran, 2004-2015: a review. East Mediterr Health J. 2017;23(3):245-256.

4. Noroozi A, Malekinejad M, Rahimi-Movaghar A. Factors influencing transition to Shisheh (methamphetamine) among young people who use drugs in Tehran: a qualitative study. J Psychoactive Drugs. 2018;50(3):214-223.

5. GBD 2016 Risk Factors Collaborators. Global, regional, and national comparative risk assessment of 84 behavioural, environmental and occupational, and metabolic risks or clusters of risks, 1990-2016: a systematic analysis for the Global Burden of Disease Study 2016". Lancet. 2017;16;390(10100):1345-1422.

6. Darke S, Kaye S, McKetin R, Duflou J. Major physical and psychological harms of methamphetamine use. Drug Alcohol Rev. 2008;27(3):253-62.

7. Zweben JE, Cohen JB, Christian D, Galloway GP, Salinardi M, Parent $\mathrm{D}$, et al. Methamphetamine Treatment Project. Psychiatric symptoms in methamphetamine users. Am J Addict. 2004;13(2):181-190.

8. Zule WA, Costenbader EC, Meyer WJ Jr, Wechsberg WM. Methamphetamine use and risky sexual behaviors during heterosexual encounters. Sex Transm Dis. 2007;34(9):689-94.

9. Ballester J, Valentine G, Sofuoglu M. Pharmacological treatments for methamphetamine addiction: current status and future directions. Expert Rev Clin Pharmacol. 2017;10(3):305-314.

10. Rawson RA, Marinelli-Casey P, Anglin MD, Dickow A, Frazier Y, Gallagher C, et al. Methamphetamine Treatment Project Corporate Authors. A multi-site comparison of psychosocial approaches for the treatment of methamphetamine dependence. Addiction. 2004 Jun;99(6):708-17.

11. Roll JM, Petry NM, Stitzer ML, Brecht ML, Peirce JM, McCann MJ, et al. Contingency management for the treatment of methamphetamine use disorders. Am J Psychiatry. 2006;163(11):1993-9. 
12. Mattick RP, Breen C, Kimber J, Davoli M. Buprenorphine maintenance versus placebo or methadone maintenance for opioid dependence. Cochrane Database Syst Rev. 2014;6(2):CD002207.

13. Nielsen S, Larance B, Degenhardt L, Gowing L, Kehler C, Lintzeris N. Opioid agonist treatment for pharmaceutical opioid dependent people. Cochrane Database Syst Rev. 2016;9(5):CD011117.

14. Hartmann-Boyce J, Chepkin SC, Ye W, Bullen C, Lancaster T. Nicotine replacement therapy versus control for smoking cessation. Cochrane Database Syst Rev. 2018;31(5):CD000146.

15. Herin DV, Rush CR, Grabowski J. Agonist-like pharmacotherapy for stimulant dependence: preclinical, human laboratory, and clinical studies. Ann N Y Acad Sci. 2010;1187:76-100.

16. Volkow ND, Chang L, Wang GJ, Fowler JS, Franceschi D, Sedler M, et al. Loss of dopamine transporters in methamphetamine abusers recovers with protracted abstinence. J Neurosci. 2001;21:9414-9418.

17. Brown JM, Yamamoto BK. Effects of amphetamine on mitochondrial function: Role of free radicals and oxidative stress. Pharmacol Exp Ther. 2003;99:45-53.

18. Pereira FC, Lourenço E, Milhazes N, Morgadinho T, Ribeiro CF, Ali $\mathrm{SF}$, et al. Methamphetamine, morphine, and their combination: Acute changes in striatal dopaminergic transmission evaluated by microdialysis in awake rats. Ann N Y Acad Sci. 2006;1074:160-173.

19. Stephans S, Yamamoto B. Methamphetamines pretreatment and the vulnerability of the striatum to methamphetamine neurotoxicity. Neuroscience. 1996;72:593-600.

20. Surmeier DJ, Ding J, Day M, Wang Z, Shen W. D1 and D2 dopaminereceptor modulation of striatal glutamatergic signaling in striatal medium spiny neurons. Trends Neurosci. 2007;30:228-235.

21. Hamada M, Higashi H, Nairn AC, Greengard P, Nishi A. Differential regulation of dopamine $\mathrm{D} 1$ and $\mathrm{D} 2$ signaling by nicotine in neostriatal neurons. J Neurochem. 2004;90(5):1094-1103.

22. Sandoval V, Riddle EL, Hanson GR, Fleckenstein AE. Methylphenidate alters vesicular monoamine transport and prevents methamphetamine-induced dopaminergic deficits. J Pharmacol Exp Ther. 2003;304:1181-1187.

23. Storebø OJ, Ramstad E, Krogh HB, Nilausen TD, Skoog M, Holmskov M, et al. Methylphenidate for children and adolescents with attention deficit hyperactivity disorder (ADHD). Cochrane Database Syst Rev. 2015;25(11):CD009885.

24. Epstein T, Patsopoulos NA, Weiser M. Immediate-release methylphenidate for attention deficit hyperactivity disorder (ADHD) in adults. Cochrane Database Syst Rev. 2016;26(5):CD005041.

25. Tiihonen J, Kuoppasalmi K, Föhr J, Tuomola P, Kuikanmäki O, Vorma $\mathrm{H}$, et al. A comparison of aripiprazole, methylphenidate, and placebo for amphetamine dependence. Am J Psychiatry. 2007;164(1):160-2.

26. Miles SW, Sheridan J, Russell B, Kydd R, Wheeler A, Walters C, et al. Extended-release methylphenidate for treatment of amphetamine/methamphetamine dependence: a randomized, double-blind, placebo-controlled trial. Addiction. 2013;108(7):1279-86.

27. American Psychiatric Association (APA). Diagnostic and Statistical Manual of Mental Disorders: DSM-IV-TR. APA Publications, 2000.

28. Mokri A. Guide on treatment of stimulant abuse based on modified matrix model. Tehran, Sepidbarg Publications, 2015. [Book in Persian]

29. McLellan AT, Kushner H, Metzger D, Peters R, Smith I, Grissom G, et al. The Fifth Edition of the Addiction Severity Index. J Subst Abuse Treat. 1992;9(3):199-213.

30. Atef-Vahid MK. Psychometric properties of fifth edition of the Addiction Severity Index in Farsi. Final report of research project. Iranian National Center for Addiction Studies (INCAS), Tehran University of Medical Sciences (TUMS), 2013. [Persian]

31. Srisurapanont M, Jarusuraisin N, Jittiwutikan J. Amphetamine withdrawal: I. Reliability, validity and factor structure of a measure. Aust N Z J Psychiatry. 1999;33(1):89-93.

32. McGregor C, Srisurapanont M, Jittiwutikarn J, Laobhripatr S, Wongtan T, White JM. The nature, time course and severity of methamphetamine withdrawal. Addiction. 2005;100(9):1320-9.

33. Ekhtiari H, Alam-Mehrjerdi Z, George S, Mokri A. Designing and evaluation of reliability and validity of visual cue-induced craving assessment task for methamphetamine smokers. Basic Clin Neurosci 2010;1:33-46.

34. Nakama H, Chang L, Cloak C, Jiang C, Alicata D, Haning W. Association between psychiatric symptoms and craving in methamphetamine users. Am J Addict. 2008;17(5):441-6.

35. Beck AT, Steer RA, Brown GK. BDI-II: Beck Depression Inventory
Manual. 2nd ed. San Antonio: Psychological Corporation, 1996

36. Ghassemzadeh H, Mojtabai R, Karamghadiri N, Ebrahimkhani N. Psychometric properties of a Persian-language version of the Beck Depression Inventory-Second edition: BDI-II-PERSIAN. Depress Anxiety. $2005 ; 21: 185-92$.

37. Family Health International (FHI). Behavioral surveillance surveys: guidelines for repeated behavioral surveys in populations at risk of HIV, 2000. Available at: http://www.fhi.org/en/hivaids/pub/guide/ bssguidelines.htm

38. Zamani S, Kihara M, Gouya MM, Vazirian M, Ono-Kihara M, Razzaghi EM, et al. Prevalence of and factors associated with HIV-1 infection among drug users visiting treatment centers in Tehran, Iran. AIDS. 2005;19(7):709-16.

39. Malekinejad M, Mohraz M, Razani N, Akbari G, McFarland W, Khairandish $\mathrm{P}$, et al. High HIV prevalence in a respondent-driven sampling survey of injection drug users in Tehran, Iran. AIDS Behav. 2015;19(3):440-9.

40. Longo M, Wickes W, Smout M, Harrison S, Cahill S, White JM. Randomized controlled trial of dexamphetamine maintenance for the treatment of methamphetamine dependence. Addiction. 2010;105(1):14654.

41. Shearer J, Wodak A, Mattick RP, van Beek I, Lewis J, Hall W, et al. Pilot randomized controlled study of dexamphetamine substitution for amphetamine dependence. Addiction. 2001;96(9):1289-96.

42. Galloway GP, Buscemi R, Coyle JR, Flower K, Siegrist JD, Fiske LA, et al. A randomized, placebo-controlled trial of sustained-release dextroamphetamine for treatment of methamphetamine addiction. Clin Pharmacol Ther. 2011;89(2):276-82.

43. Rezaei F, Emami M, Zahed S, Morabbi MJ, Farahzadi M, Akhondzadeh S. Sustained-release methylphenidate in methamphetamine dependence treatment: a double-blind and placebo-controlled trial. Daru. 2015; $15(23): 2$.

44. Ling W, Chang L, Hillhouse M, Ang A, Striebel J, Jenkins J, et al. Sustained-release methylphenidate in a randomized trial of treatment of methamphetamine use disorder. Addiction. 2014;109(9):1489-500.

45. Greenwald MK. Heroin craving and drug use in opioid-maintained volunteers: effects of methadone dose variations. Exp Clin Psychopharmacol. 2002;10(1):39-46.

46. Eap CB, Buclin T, Baumann P. Interindividual variability of the clinical pharmacokinetics of methadone: implications for the treatment of opioid dependence. Clin Pharmacokinet. 2002;41(14):1153-93.

47. Greenwald MK, Comer SD, Fiellin DA. Buprenorphine maintenance and mu-opioid receptor availability in the treatment of opioid use disorder: implications for clinical use and policy. Drug Alcohol Depend. 2014;1(0):1-11.

48. Leavitt SB. Methadone dosing and safety in the treatment of opioid addiction (special report). Addict Treat Forum. 2003 (Sept):1-8.

49. Faggiano F, Vigna-Taglianti F, Versino E, Lemma P. Methadone maintenance at different dosages for opioid dependence. Cochrane Database Syst Rev. 2003;(3):CD002208.

50. Ling W, Charuvastra C, Collins JF, Batki S, Brown LS Jr, Kintaudi $\mathrm{P}$, et al. Buprenorphine maintenance treatment of opiate dependence: a multicenter, randomized clinical trial. Addiction. 1998;93(4):475-86.

51. McGregor C, Srisurapanont M, Jittiwutikarn J, Laobhripatr S, Wongtan T, White JM. The nature, time course and severity of methamphetamine withdrawal. Addiction. 2005 Sep;100(9):1320-9. 